\title{
Abstract \\ Are pregnant mothers satisfied with the quality of care provided by government antenatal clinics? Rural Sri Lankan experience
}

\author{
Karunaratne $\mathrm{A}^{1^{*}}$, Dissanayake $\mathrm{L}^{1}$, Bandara $\mathrm{P}^{1}$, Dissanayake $\mathrm{Y}^{2}$, Agampodi $\mathrm{S}^{3}$ \\ ${ }^{1}$ MOH Office, Nuwaragam Palatha East, Anuradhapura \\ ${ }^{2}$ Teaching Hospital , Anuradhapura \\ ${ }^{3}$ Department of Community Medicine, Faculty of Medicine and allied sciences, Rajarata University of Sri Lanka
}

\begin{abstract}
Background

As a country having high coverage of antenatal care and low maternal mortality rate, in Sri Lanka, the main challenge in maternal healthcare delivery is the quality improvement. We assessed the antenatal clinic attendees' perceptions on the quality of received care as a measure of assessing the actual quality of health care delivery.

Methods

Maternal perception of the quality of antenatal care was measured through a standard questionnaire to collect information on four domains namely, "technical expertise and medication", "resources and accessibility", "interpersonal care and information" and "communication, clinic and home visits". It was administered to pregnant women above 24 weeks of POA attending government antenatal clinics in the area.

Results

Out of the interviewed 236 pregnant women, majority were Sinhalese (93.6\%) and from rural areas $(54.7 \%)$. The mean age was 28.1 years. Only $23.3 \%$ of them were employed and $74.1 \%$ were having a low monthly income. Majority (75.4\%) had at least four previous clinic visits. Most of the women were satisfied of the quality of received care. Above 93.2\% (n-220) of the participants expressed satisfaction on received medical care and above $89 \%(n=211)$ on drugs while $64.4 \%(n=152)$ were satisfied about accessibility and $61 \%(n=144)$ on indirect costs. Compassion, Respect and support shown by the staff were highly favorable to more than 55\% ( $>130$ ) of women while Information provided by the staff were favorable to $86.4 \%(n=204)$. Least satisfying factors were Long waiting time and inadequate Toilet facilities (below favorable-levels in $51.3 \%, \mathrm{n}=121$ and $50.4 \%, \mathrm{n}=120$ respectively). Rural women with low socio-economical class were more satisfied than the urban populations in all the domains of care. The main suggestion was to improve the clinics amenities.

Conclusions

Improvement of infrastructure and reducing waiting time would increase the perceived quality of care among this population. Urban rural difference in perceived care should be addressed in a different way.
\end{abstract}

Key words: Patient Satsfaction, Antenatal care, Maternal care, Sri Lanka

Copyright: $(2015$ Karunaratne A et al. This is an open access article distributed under the Creative Commons Attribution License, which permits unrestricted use, distribution, and reproduction in any medium, provided the original work is properly cited.

\footnotetext{
* Correspondence : anruddha@gmail.com
}

Cite this abstract as: Karunaratne A, Dissanayake L, Bandara P, Dissanayake Y, Agampodi S. Are pregnant mothers satisfied with the quality of care provided by government antenatal clinics? Rural Sri Lankan experience.

Anuradhapura Medical Journal 2015;9 (2Supp):S11.

DOI: http://dx.doi.org/10.4038/amj.v9i2Supp.7560 


\section{Submit your next Manuscript to}

Anuradhapura
Medical Journal

Submit your manuscript at

http://amj.sljol.info/ 